\title{
A case of craniopagus parasiticus: an antenatal diagnosis by ultrasound screening at 16 weeks of gestation and a literature review of recently reported cases
}

\author{
Houda Nasser Al Yaqoubi ${ }^{1}$, Nishat Fatema ${ }^{1}$, Badriya Sulaiman Al Fahdi ${ }^{2}$ \\ ${ }^{1}$ Department of Obstetrics and Gynaecology, Ibri Regional Hospital, Ministry of Health, Sultanate of Oman; ${ }^{2}$ Department \\ of Obstetrics and Gynaecology, Royal Hospital, Ministry of Health, Sultanate of Oman. E-mail: nishat.doc.om@gmail.com \\ Received: 2nd July 2018, Revised: 1st October 2018, 5th November 2018, Accepted: 20th November 2018
}

\begin{abstract}
SUMMARY: Al Yaqoubi HN, Fatema N, Al Fahdi BS. A case of craniopagus parasiticus: an antenatal diagnosis by ultrasound screening at 16 weeks of gestation and a literature review of recently reported cases. Turk J Pediatr 2019; 61: 941-945.

Craniopagus parasiticus (CP) is a rare type of malformation of conjoined twins, with one degenerated or underdeveloped parasite twin united at the cranium with the other fully developed twin. Only a handful of cases have been documented in the literature to date. The incidence of this rare deformity is approximately 4 to 6 out of every 10,000,000 live births. We report on a case of CP, diagnosed at 16 weeks of gestation by ultrasound screening. To the best of our knowledge, the case that we present is the first CP case that was diagnosed at such an early gestational age. The formed fetus was found to harbour complex cardiac anomalies. In view of poor prognosis of survival after delivery and upon permission from the couple, the pregnancy was terminated at 17 weeks of gestation.
\end{abstract}

Key words: twins, conjoined, craniopagus parasiticus, malformation.

Craniopagus parasiticus $(\mathrm{CP})$ is a rare type of malformation of conjoined twins, with one degenerated or underdeveloped parasite twin united at the cranium with the other fully developed twin. ${ }^{1,2}$ The term 'craniopagus' is a Greek word (kranio meaning 'head' and pagus meaning 'together'). ${ }^{3}$ Only a handful of cases have been documented in the literature to date. The incidence of this rare deformity is approximately 4 to 6 out of every 10,000,000 live births. The first reported case of this congenital malformation was Everard Home's 'Twin-headed boy of Bengal' in 1790, whose skull is preserved at the Hunterian Museum. ${ }^{1,3,4}$ Later on, only two cases have been published, where an antenatal diagnosis of $\mathrm{CP}$ was made by ultrasonography (USG) screening at 28 weeks of gestation by Okazaki et al. ${ }^{5}$ and 20 weeks of gestation by Aquino et al. ${ }^{6}$

We report on a case of $\mathrm{CP}$, diagnosed at 16 weeks of gestation by ultrasound screening. To the best of our knowledge, the case that we present is the first $\mathrm{CP}$ case that was diagnosed at such an early gestational age. The formed fetus was found to have complex cardiac anomalies. In view of poor prognosis of survival after delivery and upon permission from the couple and the ethical committee, the pregnancy was terminated at 17 weeks of gestation.

\section{Case Report}

At 16 weeks of gestation, a 19-year-old woman in her second pregnancy was referred to our fetal assessment clinic from the local health centre for a detailed anomaly scan, after detection of a mass protruding from the fetal head. The previous pregnancy of the patient had ended in the $1^{\text {st }}$ trimester by a miscarriage at 6 weeks of gestational age. The couple were consanguineous (first cousins). She was not known to have any significant previous medical or surgical comorbidity. 
During detailed ultrasound screening, the fetal medicine expert diagnosed CP. A live fetus was found to have two fully formed heads that were joined together at the right temporal area of the main cranium with one trunk and body. The patient was referred to the tertiary centre for further evaluation and counselling, and a second expert confirmed the diagnosis during ultrasound examination two days later. Fetal facial assessment showed two fully developed mouths, two noses, and four eyes with joined median orbits (Fig. 1). The fetal heart was revealed to have complex cardiac anomalies - a large atrio-ventricular septal defect with a single atrio-ventricular valve. Both pulmonary and aortic vasculature originated from the right ventricle, and the aorta was narrow with a dysplastic aortic valve.

Intracranial structures were found to be normal in both skulls according to the gestational age. A fully developed vertebral column and a single pair of upper and lower extremities were seen. The stomach bubble was small, but no significant abnormality was detected in other intraabdominal and intrathoracic organs. The liquor volume was normal, and a single placenta was placed anteriorly.
Although there are instances of survival among $\mathrm{CP}$ twins with or without surgical intervention, we counselled the family for the termination in view of poor and lethal prognosis due to complex cardiac anomalies of the formed fetus, to preempt intrauterine death at later gestation as well as the risk of a caesarean delivery for cephalo-pelvic disproportion if the pregnancy was continued till term. The family opted for the termination of the pregnancy, and one week later, the patient was admitted for medical termination of pregnancy after taking the final formal consent from the couple. On admission, she was vitally stable, with blood pressure $123 / 87 \mathrm{mmHg}$, and pulse $89 / \mathrm{min}$, baseline laboratory examinations were normal (haemoglobin: $11.5 \mathrm{gm} / \mathrm{dL}$, platelet count: $\left.233 \times 10^{9} / \mathrm{L}\right)$, and blood group was B (Rh) negative. Medical termination of pregnancy was done according to the hospital protocol with tablet misoprostol (400 microgram) given per vaginally, each tablet at four-hour intervals and up to a maximum of four doses. After receiving the 2 nd dose of misoprostol, she aborted a male fetus and its parasitic twin (Fig. 2), neither with any sign of life. After the delivery, the patient remained stable with a normal postpartum course. She received anti D (250) according to the hospital protocol.

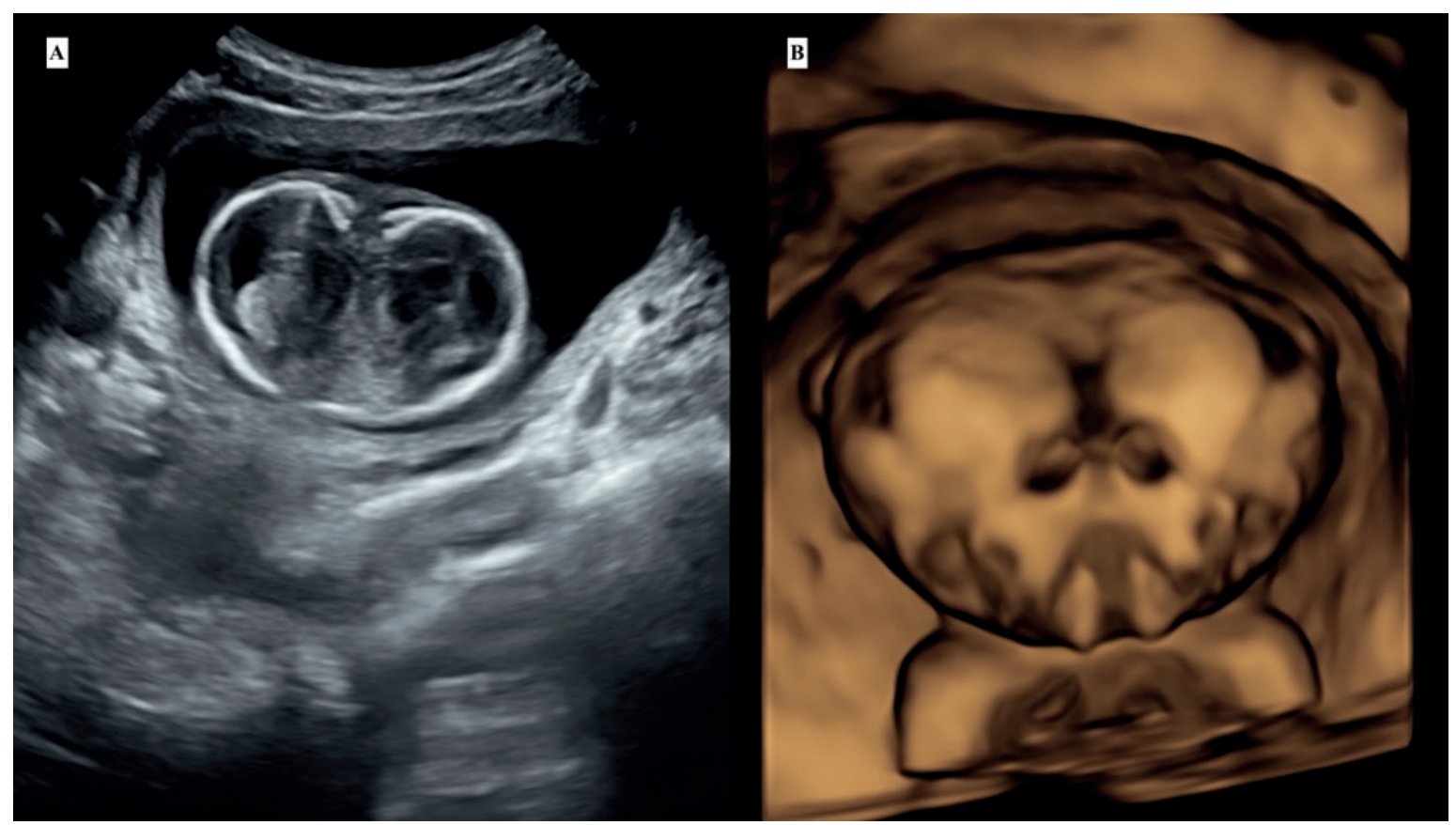

Fig. 1. A. Two-dimensional ultrasonographic view of the fetal head, arrows showing two fused craniums. B. Three-dimensional ultrasonographic view of the fetal head, arrows showing two fully developed mouths, two noses, and four eyes with joined median orbits. 


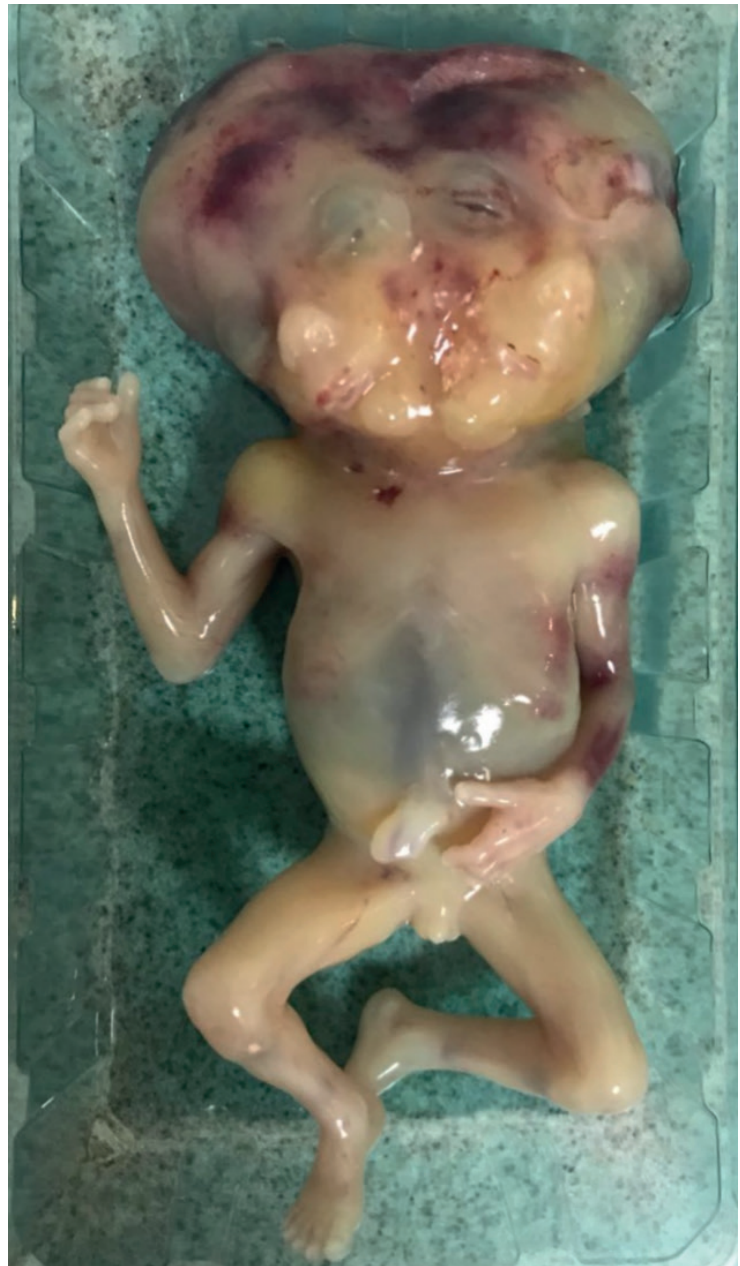

Fig. 2. Craniopagus parasiticus twin after delivery

An autopsy of the fetus was not done due to religious and cultural constraints. An informed consent was obtained from the parents for the publication and use of photographs.

\section{Discussion}

The exact aetiology for CP has not been identified. Some hypotheses or mechanisms underlying $\mathrm{CP}$ were described in previous studies, such as the 'fission theory'. This theory suggests that $\mathrm{CP}$ may result from the development of two fetuses from one zygote in the second week of gestation with incomplete separation at their cranial ends. On the other hand, it is also hypothesised that $\mathrm{CP}$ can result from the abnormal fusion of two separate embryos at the anterior open neuropore, at the end of the fourth week of gestation. Another hypothesis is that 'craniopagus parasiticus is caused by a lack of blood supply to the second twin, brought about by the degeneration of the umbilical cord, thereby preventing the development of the fetus'. ${ }^{1,4}$

CP twins share the same chorion, amnion, and placenta. In this variety of conjoined twins, the male sex predominance was reported in previous cases. ${ }^{1,4,5}$ This present case was also grossly seen to have male external genitalia. The parasitic head may be fused with its sibling at the level of the vertex, forehead or the side of the head. ${ }^{4}$ In the case we have presented, the parasitic head was attached at the right temporal area of the formed fetus.

In the present case, the couple was consanguineous. In a worldwide collaborative epidemiological study for birth defects, authors found the association of consanguinity in 5 of 209 conjoint twin cases. ${ }^{7}$

Due to the rarity of the condition, $\mathrm{CP}$ is infrequently mentioned in the literature. ${ }^{1,3}$ During the last 10 years, only a few cases have been reported. These four cases are summarised in Table I. They were either complicated with intrapartum problems or had postnatal problems due to associated anomalies.

To the best of our knowledge and after a thorough search, we found that only two cases of CP antenatally diagnosed by USG screening were documented. ${ }^{5,6}$ In 1987, Okazaki and colleagues reported the case of a 27 -year-old woman at 28 weeks of gestation presented with suspected hydrocephalous and significant polyhydramnios. After amnioreduction, a female fetus was diagnosed as CP by USG screening. Three weeks later intrauterine fetal death occurred, and a caesarean section was performed to deliver the baby. ${ }^{5}$ In another case reported by Aquino et al. ${ }^{6}$ a 25 -year-old woman was diagnosed with $\mathrm{CP}$ fetus at 20 weeks gestation (Table I). They found two bodies of discordant sized twins joined by a single cranium and the smaller of the twins with ascites and marked polyhydramnios. In view of poor prognosis, the pregnancy was terminated medically two days after diagnosis.

Early detection of this condition is desirable, since termination of pregnancy is a therapeutic 


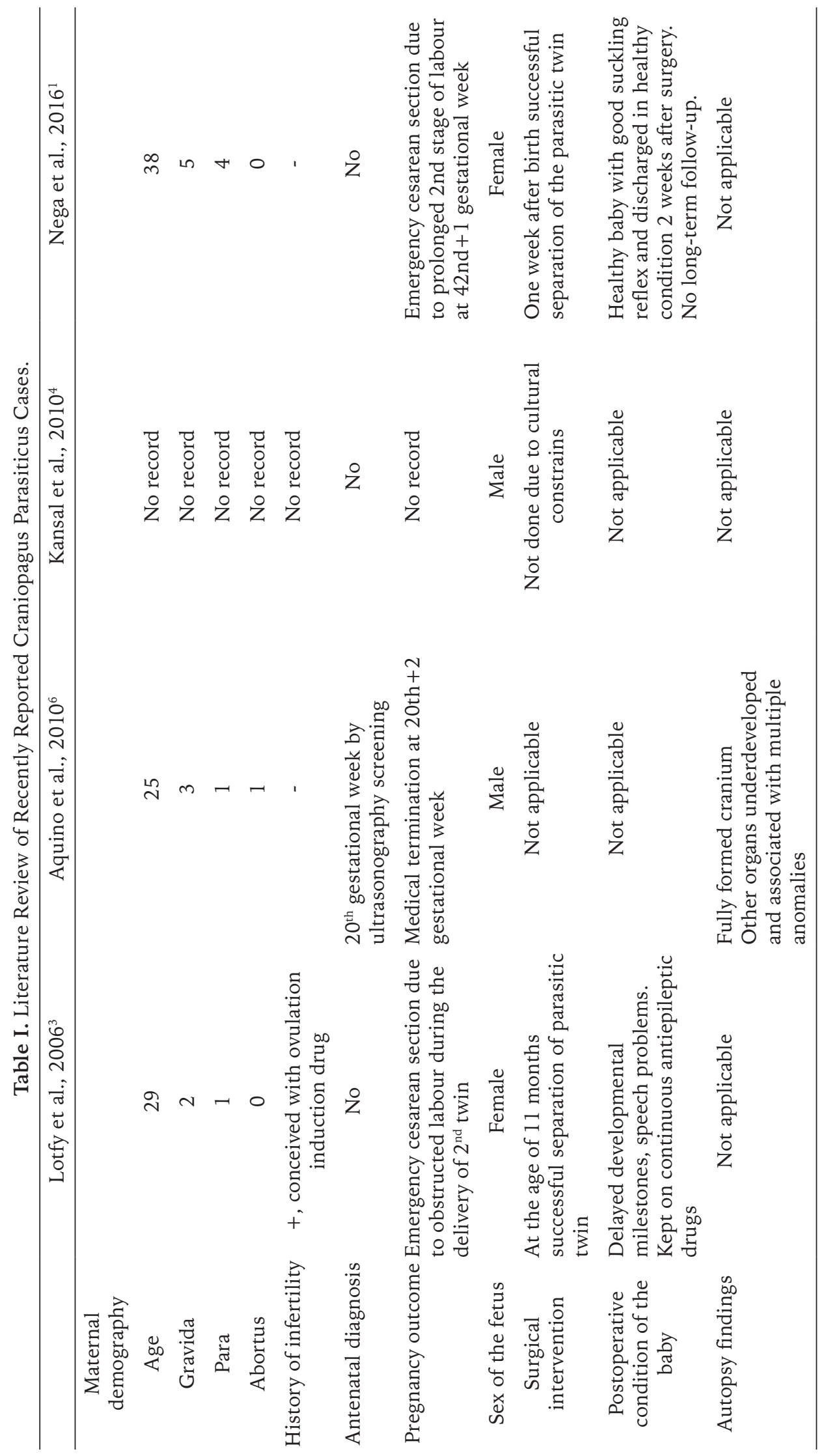


option before 24 weeks of gestation. If CP is diagnosed later, caesarean delivery remains the only option because of cephalo-pelvic disproportion. This early diagnosis of our case at 16 weeks of gestation helped us to take the prompt decision for termination of pregnancy and avoid a caesarean delivery that would have been indicated in advanced pregnancy due to cephalo-pelvic disproportion.

Very few instances of successful surgical separation of the parasitic twin from the healthy twin were mentioned in previous studies. A case has been reported by Nega et al. ${ }^{1}$ of a live-born female baby with a parasitic co-twin. Successful separation was done one week after birth. The immediate postoperative condition of the infant was good, with normal suckling reflex. Lotfy et al. ${ }^{3}$ described another similar case, where they successfully separated the parasitic twin from its co-twin at the age of 11 months. One year after surgery, the girl was alive without any neurological deficit. The catastrophic complication during surgical separation would be bleeding from the dural sinuses. Therefore, before dealing with the dural venous sinuses, the early ligation of the arterial supply to the parasite may improve the surgical outcome.

Due to the rarity of cases and limited discussions related to $\mathrm{CP}$ twins in the literature, the exact causes underlying this condition are still unknown. Although we terminated the pregnancy in view of other congenital defects and poor prognosis in our case, in salvageable cases, surgical separation is an option. ${ }^{1}$ Further observations might suggest explanations for this birth defect, which, in turn, may improve the prognosis and survival in this rare condition.

\section{REFERENCES}

1. Nega W, Damte M, Girma Y, Desta G, Hailemariam M Craniopagus parasiticus - a parasitic head protruding from temporal area of cranium: a case report. J Med Case Rep 2016; 10: 340 .

2. Bosmia AN, Smelser LB, Griessenauer CJ. An apocryphal case of craniopagus parasiticus: the legend of Edward Mordake. Childs Nerv Syst 2015; 31: 2211-2212.

3. Lotfy M, Sakr SA, Ayoub BM. Successful separation of craniopagus parasiticus. Neurosurgery 2006; 59: E1150.

4. Kansal R, Kale C, Goel A. Craniopagus parasiticus: a rare case. J Clin Neurosci 2010; 17: 1351-1352.

5. Okazaki JR, Wilson JL, Holmes SM, Vandermark LL. Diprosopus: diagnosis in utero. AJR Am J Roentgenol 1987; 149: 147-148.

6. Aquino DB, Timmons C, Burns D, Lowichik A. Craniopagus parasiticus: a case illustrating its relationship to craniopagus conjoined twinning. Pediatr Pathol Lab Med 1997; 17: 939-944.

7. Mutchinick OM, Luna-Muñoz L, Amar E, et al. Conjoined twins: a worldwide collaborative epidemiological study of the International Clearinghouse for Birth Defects Surveillance and Research. Am J Med Genet Part C Semin Med Genet 2011; 157C: 274-287. 